\title{
Sociabilidades e institucionalización del fútbol infantil en Bariloche, Argentina. La experiencia de la AFIB (1987-1994)
}

\author{
Sociabilities and the institutionalization of children's football \\ in Bariloche, Argentina. The experience of the AFIB (1987-1994)
}

\section{Luciano Arancibia Agüero*}

\footnotetext{
* Profesor de Historia (Universidad Nacional del Comahue [UNCo], Argentina). Diplomado en Investigación en Humanidades (Universidad de Buenos Aires). Becario doctoral del CONICET. Investigador en el Instituto Patagónico de Estudios de Humanidades y Ciencias Sociales (IPEHCS), sede Bariloche. Doctorando en Historia (UNCo, Bariloche).

-luciano_arancibia@yahoo.com.ar http://orcid.org/0000-0001-

7851-2135
}

RECIBIDO: 23.5.2021

ACEPTAD0: 19.7.2021

\section{Resumen}

En este artículo se reconstruye el proceso de institucionalización del fútbol infantil en la ciudad de Bariloche a partir de la creación de la Asociación de Fútbol Infantil Bariloche (AFIB), entidad que entre 1987 y 1994 se encargó de organizar y formalizar la práctica futbolística de los niños de la localidad. Mediante un análisis histórico de las interacciones sociales, se abordan las relaciones y las estrategias asociativas de los clubes barriales que integraron dicha institución. El estudio de caso permitió abordar las dinámicas institucionales, barriales y sociales de los sectores populares. Se indagaron los orígenes de la AFIB, sus objetivos, gestiones y limitaciones, así como los discretos apoyos del Estado a sus actividades. Además, se abordaron las sociabilidades motivadas por el fútbol en diversos espacios urbanos que se constituyeron como ámbitos de interacción para los vecinos. El contexto posterior al retorno de la democracia en Argentina favoreció el asociativismo deportivo, a la vez que el crecimiento urbano y la escasez de políticas públicas dificultaron la consolidación de instituciones como la AFIB. En una ciudad turística internacional, el amateurismo y el voluntarismo configuraron la sociabilidad deportiva y el desarrollo institucional.

Palabras clave: deporte, club, asociación, Argentina. 


\section{Albstract}

This article reconstructs the process of institutionalization of children's football games in the city of Bariloche, as from 1987, when the Bariloche Children's Football Association (AFIB) was founded. Between 1987 and 1994 the AFIB was responsible for organizing and formalizing football practices of local children. Social interactions, as well as the associative strategies of its members (neighborhood clubs) and their relationships are addressed through a historical analysis. The case study enabled addressing the institutional, local and social dynamics of the popular sectors. The origins of the AFIB, its objectives, efforts and limitations were investigated, as well as the moderate support offered by the State. In addition, the sociabilities motivated by football in various urban spaces established as areas of interaction for residents of the different city neighborhoods were addressed. The context after the return of democracy in Argentina favored sports associativism, while urban growth and the scarcity of public policies hindered the consolidation of institutions such as the AFIB. In an international tourist city, amateurism and voluntarism shaped neighborhood sports sociability and institutional development.

Keywords: sports, club, association, Argentina.

\section{Introducción}

Argentina es por excelencia un país futbolero. El fútbol es un fenómeno que moviliza a miles de aficionados y practicantes en todos los rincones del país. Junto con el fanatismo que suscitan la selección nacional, los héroes deportivos y los equipos profesionales, el fútbol también es una práctica que desarrollan en la cotidianidad miles de personas en la calle, plazas, clubes y otros espacios, de manera amateur o informal. Sin embargo, no abundan los estudios que analicen en la historia reciente las sociabilidades y los vínculos generados en torno a la práctica futbolística en los clubes vecinales. En general, los escritos sobre la relación entre deporte, sociedad y política se han centrado en el abordaje de los principales clubes de las ciudades más grandes del país. Por fuera de las áreas centrales, es exigua la bibliografía en las regiones del interior de la Argentina, y mucho más si se trata de la actividad barrial.

La ciudad de San Carlos de Bariloche no escapa a este panorama, ya que existen pocos trabajos que aborden las relaciones y las sociabilidades deportivas construidas por los sectores populares en las últimas décadas. Una excepción es el estudio de Javier Nestares (2017), quien se adentra en un caso de organización en torno al asociacionismo deportivo —el proceso de creación y consolidación de la Asociación de Deportes y Fútbol Libre de 
Bariloche, fundada en 1986 como la principal entidad dedicada al fútbol amateur en la ciudad - y lo hace como medio para analizar algunas tensiones en la sociedad local.

Bariloche es una ciudad turística de tamaño intermedio ubicada en la zona andina del norte de la Patagonia, al oeste de la provincia de Río Negro. En este artículo vamos a reconstruir el proceso de institucionalización del fútbol infantil en esta localidad a partir de la creación de la Asociación de Fútbol Infantil Bariloche (AFIB), entidad que desarrolló sus actividades entre 1987 y 1994. Esta institución, emergida en los años posteriores al retorno de la democracia en Argentina, en 1983, se encargó de organizar la práctica deportiva de los niños varones de la localidad y otorgarles un marco formal a los encuentros que ya se venían realizando de manera inorgánica en distintos espacios públicos de la ciudad.

Se trata de una de las primeras instituciones dedicadas al fútbol infantil en el país, cuyas actividades dinamizaron los vínculos vecinales y la integración social en un contexto marcado por la intensificación de los procesos de segregación socioespacial en Bariloche. Su experiencia nos lleva a pensar las particularidades locales de la sociabilidad deportiva y el desarrollo de las instituciones frente a los procesos de globalización y mercantilización del deporte a escala mundial y en las grandes ciudades de la Argentina. En una ciudad turística internacional, el devenir del fútbol local se mantuvo al margen de los procesos de comercialización del deporte-espectáculo, la popularización masiva de la actividad y la compraventa de jugadores desde edades tempranas con motivo de su inserción en el circuito competitivo.

Abordaremos el devenir de la Asociación, así como también las relaciones construidas por los clubes que formaron parte de ella, en su dimensión histórica y espacial. De esta forma, planteamos una reflexión acerca del desarrollo de las instituciones populares en Bariloche desde una perspectiva que vincula las sociabilidades deportivas con las dinámicas sociales y vecinales entre los años posteriores al retorno de la democracia y la irrupción del neoliberalismo, en los años noventa. Indagamos en la conformación de la AFIB, sus objetivos, gestiones y limitaciones, así como también en la lógica institucional, las formas de participación de sus miembros y las redes tejidas con otras entidades. Como parte de ello, exploramos los apoyos estatales al desarrollo del fútbol infantil y tratamos las sociabilidades motivadas por la actividad en diversos espacios urbanos que se constituyeron como ámbitos de interacción vecinal.

En los años ochenta y noventa, la ciudad de Bariloche tuvo un crecimiento acelerado y desorganizado que trajo consigo un aumento de la segregación socioespacial, al calor de las crisis económicas, la aplicación de políticas neoliberales y las dificultades del Estado municipal para brindar servicios públicos a las zonas periféricas. El contexto de la reapertura democrática y las nuevas configuraciones barriales favorecieron la emergencia de experiencias asociativas como la conformación de clubes de fútbol. Sin embargo, las últimas décadas del siglo XX son consideradas una etapa de decadencia de 
esas instituciones vecinales en Argentina, como resultado de la valorización del paradigma del individualismo y los postulados liberales de la economía (Zambaglione et al., 2013). En definitiva, proponemos aportar, a partir del estudio de las sociabilidades deportivas y urbanas, un abordaje local — desde una región alejada de las áreas centrales del país - que complejice y contribuya a comprender las realidades de los clubes barriales en Argentina en el contexto finisecular.

El recorte temporal abarcado por la vida institucional de la AFIB es un período contradictorio, que permite ver las potencialidades de los clubes para generar integración social a través del asociacionismo, pero que también deja al descubierto sus limitaciones. El crecimiento de la ciudad y la escasez de políticas públicas coartaron las posibilidades de consolidación material de las organizaciones populares barilochenses, en su mayoría regidas por el amateurismo en sus gestiones y el desarrollo de sus actividades a pulmón. Estas características del contexto nos llevan a explicar las estrategias de funcionamiento de dicha asociación. Por eso, analizamos las relaciones que los clubes tejieron con otras organizaciones en los ámbitos barrial, local e incluso fuera de la ciudad. Las entidades deportivas creadas en esos años construyeron un entramado de redes sociales que dieron paso a vínculos relativamente igualitarios y a acciones que motivaron la inclusión vecinal. Asimismo, fomentaron la participación de los sectores populares en el espacio público y generaron mecanismos para intentar desarrollar sus estructuras institucionales en un período atravesado por las crisis y la fractura social.

Este artículo da cuenta de algunos avances empíricos de investigación realizados en el marco del plan de trabajo titulado «Políticas públicas, sociabilidades urbanas y fútbol: los clubes barriales en el oeste rionegrino (1970-2019)», propuesto para el desarrollo de una beca doctoral otorgada por el Consejo Nacional de Investigaciones Científicas y Técnicas de Argentina para el período 2020-2025.

\section{Consideraciones teórico-metodológicas}

Desde un abordaje de la historia social, buscamos indagar en las formas de sociabilidad de una población local determinada y reconstruir las relaciones establecidas entre los actores implicados. El estudio de las interacciones, en su dimensión histórica y espacial, resulta fundamental para comprender la complejidad de los procesos sociales y analizar las particularidades de una asociación. A su vez, consideramos los contextos locales y nacionales que atraviesan la realidad abordada, para entender de manera situada las relaciones y acciones de dichos actores.

El concepto de sociabilidad permite abordar las interacciones de la vida popular en el tiempo libre y sus expresiones en un marco espacial y temporal concreto. Maurice Agulhon lo definió como «el campo que integra las relaciones interindividuales que se 
desarrollan en el seno de los grupos intermedios [...], aquellos que se insertan entre la intimidad del núcleo familiar y el nivel más abstracto de las instituciones» (Escalera, 2000, p. 2). Además, Josepa Cucó i Giner afirma: «A nivel local, las relaciones y prácticas de sociabilidad conforman redes y grupos sociales que son dinámicos y variables y se hallan inscritos en las formas de vida cotidiana» (2008, p. 66). La sociabilidad deportiva en los clubes facilita la constitución de la base social de ciertos espacios urbanos que actúan como ámbitos unificadores y creadores de identidades populares (Navarro, 2006). En estos espacios, las relaciones se articulan en niveles como el familiar y el vecinal, donde la proximidad geográfica y la interacción cotidiana suscitan el encuentro en la calle, canchas y otros sitios.

Entendemos los clubes como espacios de sociabilidad, generadores de un complejo entramado de interacciones que dan paso a vínculos identitarios y acciones colectivas alrededor de la práctica futbolística. El campo de la sociabilidad deportiva y sus instituciones posee redes complejas que nos llevan a indagar en las particularidades de la creación y el desarrollo de las asociaciones. ${ }^{1}$ Además, tenemos en cuenta que el deporte trae consigo la producción de identidades, discursos y moralidades construidos al calor de las relaciones en las instituciones (Hang, 2018). Por otra parte, la sociabilidad formal que tiene lugar a través de los clubes, generalmente con una clara intencionalidad, se encuentra estrechamente ligada al desarrollo de una sociabilidad informal, de carácter más espontáneo, que se da en la cotidianidad por fuera del encuadre institucional, en el hogar, la calle, cafés, bares, entre otros espacios. En este artículo nos centramos en el estudio de la vida asociativa, pero sin dejar de lado las interacciones sociales no regladas, teniendo en cuenta que las expresiones formales e informales de la sociabilidad pueden confluir y complementarse.

Cuando hablamos de deporte amateur — dentro del cual ubicamos la organización del fútbol barrial barilochense - nos referimos a aquel que está dirigido al uso recreativo del tiempo libre, el mantenimiento de la salud y la construcción de vínculos sociales en espacios participativos que propician la integración social (Nestares, 2017, pp. 16-17). Este tipo de práctica se diferencia del deporte espectáculo, orientado al profesionalismo y el alto rendimiento, en el que se hacen presentes intereses económicos y políticos que trascienden las actividades físicas. En este último caso, el deporte deja de ser un ejercicio lúdico para convertirse en una mercancía que forma parte del entretenimiento mediático.

Empleamos el estudio de caso (Neiman y Quaranta, 2006) como método para explorar en profundidad la realidad particular de la AFIB, pero apuntando a comprender

Según Josepa Cucó i Giner, un campo de sociabilidad es «un espacio de sociabilidad específico, dotado de relativa homogeneidad y congruencia a nivel interno, cultivado por agentes sociales que comparten algún o algunos elementos significativos (ideología, etnicidad, género, etcétera), que puede llegar a generar algún tipo de identidad compartida aprehendido como tal por el conjunto social» (2008, p. 78). 
cuestiones de mayor alcance, como la problemática del crecimiento urbano y las consecuencias del neoliberalismo sobre los sectores populares en Bariloche. Como hemos expresado, la elección de la AFIB se debe al hecho de que sus actividades dinamizaron las interacciones entre vecinos de diferentes barrios, y la construcción de redes deportivas dio paso a vínculos y acciones orientados a generar integración social en un contexto de intensificación de los procesos de segregación socioespacial, a finales del siglo XX. En este punto es importante observar la repercusión que las transformaciones sociales, económicas y culturales del período tuvieron sobre las formas de sociabilidad.

Recurrimos al concepto de segregación socioespacial para caracterizar el mosaico barrial de Bariloche. La distribución desigual de la población por su condición social y económica trae consigo oportunidades dispares de acceso a los recursos. Para Fernando Groisman (2010), la segregación sitúa a los distintos grupos en un contexto de interacción uniforme, lo que puede llevar a naturalizar las diferencias y favorecer la desintegración social. Además, la concentración de la pobreza en determinadas zonas de la ciudad (generalmente periféricas) puede conducir al aislamiento social de algunos territorios y la reducción de oportunidades de integración de los más vulnerables.

Concretamente, tratamos la estrecha relación de los clubes de la AFIB y su entorno barrial, mediada por la sociabilidad deportiva. Tanto las instituciones como los barrios son construcciones sociales, productoras de realidades materiales y culturales que dan lugar a significaciones y lazos identitarios como resultado del devenir histórico de una sociedad determinada (Ignacio, 2013). El anclaje territorial vecinal resulta fundamental en el análisis de las relaciones espacializadas, así como también el marco sociohistórico en el que se desenvolvieron y la configuración de identidades.

En esta reconstrucción incorporamos diversos documentos de la AFIB que nos fueron cedidos por exdirigentes del fútbol infantil. Las colecciones incluyen actas de reuniones de la Asociación, correspondencia, notas, estatutos y artículos de la prensa local y regional. Además, presentamos información extraída de testimonios de dichos actores, los cuales nos permitieron interiorizarnos en la institucionalización del fútbol infantil y las diferentes instancias de sociabilidad alrededor de la práctica deportiva.

\section{Antecedentes}

A lo largo de la mayor parte del siglo XX, la actividad del fútbol infantil en Bariloche se desarrolló de manera fragmentada, por iniciativas dispersas de algunos clubes. Distinto es el caso del fútbol para mayores, que tuvo su proceso de institucionalización más temprano, a finales de los años treinta, con la creación de la Asociación de Football de Bariloche (Chiappe, 2019). Esa fue la primera organización rectora de la actividad en la localidad. Años más tarde, en 1952, se fundó la Liga de Fútbol Bariloche (LIFUBA), que 
aún hoy es la entidad encargada de organizar el fútbol federado en la ciudad, afiliado a la Asociación de Fútbol Argentino (AFA).

Si bien el fútbol infantil local careció de un encuadre similar durante la mayor parte de dicha centuria, podemos identificar cuatro etapas de impulso a la actividad previas a su institucionalización, concretada a mediados de los años ochenta. La primera de ellas tuvo lugar durante el peronismo (1946-1955), a partir de los Campeonatos Infantiles y Juveniles María Eva Duarte de Perón en todo el país, que tuvieron cita en Bariloche desde 1949. En ese momento, el impulso estuvo dado por la iniciativa del Gobierno nacional, con los objetivos de propiciar el «deporte social», fomentar la salud y democratizar el acceso de toda la población a las actividades físicas. Según Raanan Rein, el peronismo concebía el deporte como «una herramienta para enarbolar valores y conceptos identificados con el justicialismo [...] y, en particular, un polo de atracción para niños y jóvenes, la futura generación del país y del movimiento» $(2015$, p. 11). Dichos certámenes congregaban a equipos representativos de escuelas y de barrios, y los partidos se disputaban en terrenos baldíos en los cuales se improvisaban canchas. Sin embargo, la realización de los Campeonatos Evita se vio interrumpida por el golpe de Estado que derrocó a Perón en 1955.

La segunda etapa de impulso al fútbol infantil se dio por iniciativa de la Iglesia católica entre 1959 y 1973, específicamente a partir de los torneos organizados de forma amateur por el cura salesiano Juan Carlos Videla, que se realizaban en distintos espacios públicos de Bariloche. ${ }^{2}$ Estos encuentros convocaban a niños provenientes de distintos puntos de la ciudad, que jugaban representando a clubes federados o a equipos barriales. La asistencia a misa era un requisito obligatorio para participar, lo que da cuenta de una estrategia empleada por la Iglesia para ganar adeptos entre los sectores populares a través de la práctica deportiva (Nestares, 2017).

A pesar de aquel uso instrumental, el fútbol fue un medio que fomentó la inclusión social y espacial en una ciudad que comenzaba a expandirse hacia el sur, pero también en las zonas oeste y este, a medida que se consolidaba como centro turístico. A partir de los años sesenta, la localidad experimentó un acelerado crecimiento demográfico, a la par del aumento de la superficie del ejido municipal y la falta de planificación del espacio urbano por las autoridades comunales. Este crecimiento dio lugar a un proceso de diferenciación espacial de la población de acuerdo con su origen y su condición socioeconómica (Matossian, 2014). También como resultado del devenir de la ciudad, una parte importante de las áreas construidas en la zona urbana se caracterizaron por estar separadas por amplios terrenos baldíos, muchos de los cuales fueron acondicionados como

2 Comunicaciones personales (Julián, exdirigente del Club Las Mutisias, Bariloche, 26 de enero de 2018; Alfredo, exdirigente del Club Alas Argentinas, 25 de agosto de 2017; Guido, exdirigente del Club San Francisco II y III, 1 de noviembre de 2017; Juan Carlos B., exdirigente del Club Tiro Federal, 17 de agosto de 2017). 
campos de juego. Algunos de estos espacios fueron sede de los campeonatos del padre Videla, a partir de los cuales comenzaron a ser estrechos los vínculos entre la Iglesia y el fútbol en los barrios populares.

El siguiente momento estuvo dado por el breve retorno de los Juegos Nacionales Evita durante los años del tercer peronismo, entre 1973 y 1975. Por último, la cuarta etapa tuvo lugar durante la última dictadura cívico-militar, por la iniciativa simultánea de la Gendarmería Nacional y los actores barriales ligados al fútbol local. Del seno de dicha fuerza surgió la Gendarmería Infantil, que constituyó un intento del régimen militar por intervenir en la educación y la socialización de los niños y jóvenes en las áreas fronterizas de la Argentina. Específicamente buscaba inculcar valores militares y una identidad nacional, así como estrechar los vínculos de la población con las fuerzas de seguridad. En Bariloche cerca de mil niños se inscribieron en los escuadrones de la Gendarmería Infantil, cuya conformación incluyó la creación de un club (Lvovich y Rodríguez, 2011). Más allá de esta institución, los clubes federados y los equipos barriales también fueron responsables del desarrollo de la actividad del fútbol infantil a finales de los años setenta y principios de los ochenta, independientemente de los intereses de la dictadura, contrarios a la organización colectiva.

Vale aclarar que lo relatado hasta aquí no corresponde a un proceso lineal, sino a uno lleno de avances y retrocesos y que estuvo a cargo de diferentes actores (el Gobierno peronista, la Iglesia, la Gendarmería y los clubes). Si bien son etapas consecutivas, sería arriesgado afirmar que hubo una continuidad que derivó en la institucionalización del fútbol infantil barilochense. Más allá de este desarrollo a lo largo de las décadas, no fue hasta finales del siglo XX que la actividad comenzó a organizarse de manera sistemática.

A comienzos de la década de 1980, primaban en la localidad los torneos improvisados por grupos de amigos o vecinos que creaban equipos barriales de niños para medirse en diversas canchas de la ciudad. La mayoría de los clubes existentes (formales o informales) contaban con pocas categorías deportivas o apenas lograban juntar jugadores para conformar un solo equipo, generalmente integrado por niños de diferentes edades. Por ese entonces, la Liga de Fútbol Bariloche solía convocar a equipos barriales a disputar torneos infantiles, pero se trataba de iniciativas poco sistemáticas.

\section{La Asociación de Fútbol Infantil Bariloche: fundación, objetivos y sociabililidades}

A partir del retorno de la democracia en Argentina, en 1983, el Gobierno Nacional difundió un discurso de defensa de la institucionalidad y la participación ciudadana en 
el espacio público. Dicho discurso permeó distintos ámbitos de la sociedad civil y favoreció la emergencia de diversas experiencias asociativas. En este marco ubicamos el proceso de institucionalización del fútbol para niños en Bariloche. Las primeras actas que hallamos de la AFIB son anteriores a su fundación formal. Si bien las fuentes datan de los primeros meses de 1986, podemos inferir, a partir de los testimonios y del grado de desarrollo descripto en los documentos, que la organización ya se venía gestando en los años anteriores.

En concreto, la fundación se consumó el 7 de marzo de 1987, en una reunión de delegados representantes de ocho clubes locales realizada en las instalaciones del Estadio Municipal de Bariloche. En ese encuentro quedó conformada de común acuerdo la comisión directiva de la organización y se determinó el cobro de una cuota mensual a los clubes para el mantenimiento de la nueva asociación y para llevar adelante las actividades deportivas (Asociación del Fútbol Infantil Bariloche [AFIB], 1987a, 1987b). Algunos de estos clubes formaban parte del fútbol federado de LIFUBA con sus categorías mayores, mientras que otros eran equipos vecinales, representativos de colectividades o de instituciones tradicionalmente dedicadas a otros deportes. La AFIB se asentó sobre la práctica futbolística barrial que ya estaba instalada en Bariloche a través de encuentros más o menos espontáneos de niños y adultos en distintos espacios públicos.

Desde su fundación, la AFIB manifestó su interés por el desarrollo de una función formativa y de contención social con los niños alrededor de la práctica deportiva. Este objetivo quedó plasmado en el primer artículo de su reglamentación estatutaria:

Esta asociación es creada con el propósito de fomentar la práctica del fútbol infantil en la niñez barilochense y la zona, en forma organizada, y, de esta manera, coadyuvar en la formación del niño, a su normal crecimiento en lo físico y mental, sacándolo de malos propósitos e ideas tergiversadas y además desvinculándolo de los juegos callejeros, electrónicos, y del flagelo de las drogas que tanto acechan en la actualidad a la niñez y adolescencia en general. Esta actividad estará vinculada estrechamente con el comportamiento en la vida común familiar y obviamente escolar, no persiguiendo fines de lucro. (AFIB, 1987c)

La propuesta de fomentar la formación física y moral de los niños a través de la práctica futbolística partía de un diagnóstico realizado por la dirigencia de la AFIB en relación con las problemáticas que afectaban a las familias de los barrios populares de Bariloche. Estos se encontraban en un proceso de crecimiento, a la par del aumento de la marginalidad de amplios sectores de la población. Entre las problemáticas recordadas por los miembros de la Asociación se destacan el desempleo, la pobreza, la mala alimentación, el alcoholismo y la violencia. En numerosos testimonios se repite la idea de «sacar a los niños de la calle» e iniciarlos en el deporte. Además, los dirigentes buscaron 
inculcar la importancia de la formación en valores a través del fútbol, especialmente en su fase recreativa. ${ }^{3}$ Para ello, la AFIB intentó establecer vínculos con las familias de los niños y con las instituciones educativas a las que asistían.

La organización obtuvo su personería jurídica en mayo de 1988, otorgada por el gobierno de la provincia de Río Negro, y contó con reconocimiento del Consejo Federal del Fútbol Argentino. Con este encuadre formal, uno de los requisitos demandados a los clubes para integrar la AFIB era contar con personería jurídica. A diferencia de LIFUBA, la Asociación no exigía a los equipos poseer una cancha, una sede o determinado patrimonio, lo que permitió la participación de entidades que no tenían infraestructura ni fuentes de recursos propios. ${ }^{4}$

La emergencia de los clubes vecinales que conformaron la AFIB se produjo en el marco de un proceso de organización comunitaria en los barrios durante los años ochenta, motivado por el crecimiento de la ciudad y favorecido por la restauración democrática. Durante la segunda mitad del siglo XX, Bariloche creció de manera acelerada, desorganizada y regida por la lógica del mercado de tierras. Esto trajo consigo dificultades al Estado municipal para brindar servicios públicos a las áreas urbanas periféricas. Sumado a ello, el desarrollo del turismo como principal actividad económica, el crecimiento demográfico y la heterogeneidad social contribuyeron a la conformación de una ciudad con marcadas desigualdades socioterritoriales.

Desde finales de la década de 1980 surgieron numerosas juntas vecinales que buscaron hacer frente a las demandas que el Estado no podía satisfacer. Este tipo de institución se erigió «como unidad primaria de participación y principal articuladora en las relaciones, más o menos conflictivas, entre vecinos y el gobierno local y también entre los distintos barrios entre sí» (Matossian, 2016, p. 65). Simultáneamente, emergieron otras experiencias asociativas en los barrios, como clubes, bibliotecas populares e instituciones educativas de gestión social. Las nuevas entidades no solo tenían el objetivo de tramitar y administrar la provisión de servicios y de infraestructura, sino también de cubrir las necesidades educativas y recreativas de una población en crecimiento que demandaba espacios de socialización y de participación ciudadana. De este modo, se trató de un contexto propicio para la creación de clubes de fútbol. En torno a las nuevas instituciones se reforzaron los vínculos entre los vecinos y se crearon lazos de identificación e interacción. Ligadas a ello, la construcción de las identidades deportivas y la configuración de los barrios fueron procesos simultáneos y con influencia recíproca. julio de 2017; Juan Carlos T., exdirigente del Club Alas Argentinas, 3 de noviembre de 2017; Gervasio, exdirigente del Club Las Mutisias, 18 de agosto de 2017; Miguel, exdirigente del Club Martín Güemes, 3 de agosto de 2017). 
Por su parte, la dirigencia de la AFIB sostuvo un discurso que resaltó las bases democráticas sobre las que se asentó la organización, tanto en la participación de sus miembros como en los procesos de toma de decisión. Como parte de este discurso se dio la apertura a todos los clubes de la localidad que quisieran formar parte de la Asociación con sus categorías infantiles. Además, se enviaron invitaciones a equipos de los poblados cercanos a Bariloche, como Villa Traful, Villa La Angostura, Ñorquinco, El Bolsón, Ingeniero Jacobacci, Pilcaniyeu, Comallo y Piedra del Águila (AFIB, 1987b). ${ }^{5}$ Esta apertura respondía al interés por fortalecer a la institución y propiciar su crecimiento.

La organización de los campeonatos estaba regida por los reglamentos de alcance nacional. Además, la Asociación buscó regular la conformación de los equipos en categorías por edades. En las actas de la AFIB pueden observarse las discusiones en torno a los reglamentos, la organización de torneos, los pases de los jugadores y los regímenes de sanciones. No obstante, sobre todo en los momentos fundantes, muchas decisiones del quehacer cotidiano fueron informales, a través de acuerdos entre los clubes asociados.

Concretamente, la AFIB se encargó de organizar campeonatos al aire libre durante la época de verano, los que se disputaban en distintas canchitas distribuidas por toda la ciudad. De igual modo, en la temporada invernal propició la realización de certámenes en gimnasios techados pertenecientes a escuelas y otras entidades públicas o privadas. La organización de las jornadas deportivas se daba de manera colaborativa, mediante la división de tareas entre todos los clubes. Esto significaba un cambio respecto de la dinámica de los años anteriores a la creación de la AFIB, cuando los campeonatos eran llevados adelante por la iniciativa aislada de algunos equipos.

Los certámenes de fútbol infantil conllevaban importantes desplazamientos de población, lo que favorecía la integración de vecinos de barrios alejados entre sí alrededor de una actividad que los congregaba. En los testimonios orales son frecuentes los relatos acerca de los dirigentes y técnicos que llevaban sus equipos caminando o en vehículos particulares hasta las canchas en las que tenían pactados sus partidos. ${ }^{6} \mathrm{El}$ traslado de los equipos desde zonas periféricas de la ciudad - muchas veces marcadas por las condiciones socioeconómicas desfavorables - hacia zonas más «céntricas» resulta significativo porque implicaba para los actores involucrados una apropiación y una producción del espacio público de la ciudad a través de los encuentros deportivos que propiciaban su participación, integración y visibilización. Las fuentes dan cuenta del voluntarismo y el amateurismo con que eran llevadas adelante las actividades del fútbol infantil. Estas características imprimieron su sello en la configuración de la sociabilidad deportiva barrial y en el desarrollo de la AFIB.

6 Comunicaciones personales (Miguel, 3 de agosto de 2017; Gervasio, 18 de agosto de 2017; Pablo, exdirigente del Club Alas Argentinas, 12 de diciembre de 2017). 
Por otra parte, las actividades cotidianas de la Asociación y de los clubes implicaban la realización de reuniones asamblearias o de comisión directiva, junto con otras de carácter más informal en ámbitos públicos o privados (en canchas, la calle, viviendas). En estas instancias se intensificaron los vínculos entre los miembros de los clubes, pero también con otras entidades con las que se compartían intereses, como juntas vecinales, escuelas y comercios. La relación entre las instituciones deportivas no se limitó solo al momento concreto de los encuentros en canchas y gimnasios; también incluía reuniones de delegados, intercambios de correspondencia y organización de eventos en conjunto. Asimismo, la actividad institucionalizada dio lugar a otro tipo de relaciones de carácter más informal y espontáneo, favorecidas por la afinidad y los vínculos de vecindad. Así, el fútbol trascendía el ámbito de los clubes y llegaba a distintas canchitas, donde la práctica era realizada de forma recreativa por amigos, vecinos y conocidos.

\section{Problemáticas organizativas y materiales de la AFIB}

Durante sus años de existencia, la AFIB experimentó dificultades de índole organizativa y material que dificultaron su consolidación institucional. Así, por ejemplo, fueron frecuentes los problemas para conseguir árbitros que rigieran los partidos de la Asociación, debido a los costos que eso demandaba (AFIB, 1989, 1990). Sobre todo en los primeros años, también hubo discusiones en torno a la conformación de las categorías, ya que en ocasiones los equipos no respetaban las edades de los jugadores (AFIB, 1987d). Otro inconveniente era que algunos conjuntos no se presentaban a jugar sus partidos por desentendimientos logísticos, por mal estado de las canchas o por las largas distancias que algunos planteles debían recorrer hasta otros puntos de la ciudad (Bariloche tiene uno de los ejidos urbanos más extensos del país). ${ }^{7}$

El trabajo de las dirigencias del fútbol infantil y de los socios de los clubes tenía un fuerte componente de voluntarismo. Es decir, las estructuras asociativas eran sostenidas gracias a la unión de las voluntades de personas vinculadas por redes de solidaridad y cooperación que llevaban adelante tareas (en comisiones, subcomisiones $\mathrm{u}$ otros espacios) de manera ad honorem, destinando su tiempo y esfuerzo en beneficio de las organizaciones. Frecuentemente los mismos individuos desempeñaban varias funciones a la vez: dirigentes, delegados, directores técnicos. Muchos formaron parte de la organización de los clubes en tanto sus hijos participaban como jugadores, pero 
luego abandonaban la actividad, lo que comprometía la continuidad de los proyectos institucionales.

En cuanto a los clubes, la mayoría no contaba con campo de juego propio, elementos deportivos ni sede social. En estos casos, las reuniones de asambleas y de comisión directiva se celebraban en viviendas particulares o en espacios prestados por escuelas, juntas vecinales u otras instituciones. Por otra parte, la única fuente de ingresos de la Asociación eran las cuotas mensuales que recaudaba entre los clubes, y en las actas son recurrentes los pedidos de los directivos para que las entidades deportivas saldaran sus deudas con la AFIB. ${ }^{8}$ La situación deficitaria solía perdonarse a los clubes más necesitados para permitir que los niños pudieran continuar realizando actividades deportivas. ${ }^{9}$ De este modo, las instituciones manejaban muy poco dinero y los recursos disponibles se empleaban para las necesidades más inmediatas. El resultado de este modelo de gestión - con administraciones tan humildes - fue el poco crecimiento estructural de los clubes.

Los campeonatos de la AFIB se desarrollaron en diversas canchas y potreros distribuidos a lo largo de toda la ciudad. La mayoría de los campos de juego eran terrenos baldíos ubicados en barrios populares o periféricos de Bariloche. Solo unas pocas eran canchas sembradas pertenecientes a clubes - los predios de Alas Argentinas, Estudiantes Unidos y Don Bosco- y a ellas se sumaba el Estadio Municipal, que era cedido por las autoridades comunales para disputar las competencias más importantes. Las canchas barriales y los gimnasios de la ciudad se constituyeron como espacios de sociabilidad y de integración que congregaban a vecinos de distintos sectores de Bariloche. Sin embargo, la proliferación de potreros no tuvo correspondencia con el estado de los campos de juego, que era precario en términos generales. La mayoría estaban emplazados sobre terrenos mallinosos ${ }^{10}$ e inundables, de difícil mantenimiento, a lo que se agregaba la falta de infraestructura.

Por otra parte, la mayoría de los entrenadores y dirigentes carecían de formación específica y de discursos consensuados acerca de las tareas de contención social desarrolladas con los niños. Hasta finales de los años ochenta no había directores técnicos profesionales en Bariloche. Un hito importante para revertir parcialmente esta situación fue la realización de un curso organizado por la Asociación de Técnicos del Fútbol Argentino (ATFA) que tuvo lugar en la ciudad entre 1990 y 1991, con apoyo del gobierno municipal. El curso, válido como título terciario, significó un salto adelante en la capacitación de los técnicos. Sin embargo, la puesta en práctica de las mejoras pedagógicas y técnicas encontró su límite en las carencias materiales de los clubes.

$8 \quad$ AFIB (1987i, 1987e, 1987j).

9 Comunicación personal (Luis, dirigente de la AFIB, 23 de octubre de 2017).

10 N. del e.: Mallín es una zona de pradera inundable o cenagosa propia de la región semidesértica de la Patagonia. 


\section{Redes de sociabilidad en el fútbol infantil}

Frente a las situaciones de severa precariedad material, los clubes integrantes de la AFIB llevaron adelante distintas iniciativas para recaudar fondos, lo que formaba parte importante del desarrollo de la sociabilidad deportiva y popular en torno al fútbol infantil. La mayoría de las instituciones dio lugar a la creación de comisiones de padres, las cuales trabajaban en conjunto con las dirigencias para generar recursos que permitieran sostener la cotidianidad de los clubes (Club Don Bosco, 1989; Club 9 de Julio, 1990). En concreto, estas comisiones organizaron rifas, ventas de comida y eventos de diversa índole. Todas estas actividades contaron con la participación de los vecinos de las áreas de influencia de cada club. Los testimonios y los documentos dan cuenta de la notable participación de mujeres en los equipos de trabajo, principalmente familiares de los niños jugadores, quienes además ocuparon algunos puestos en las comisiones directivas de los clubes (pero no de la AFIB). ${ }^{11}$

De la mano de dichas actividades, los clubes y las comisiones de madres y padres fomentaron otro tipo de reuniones que trascendían lo deportivo y que estaban orientadas a motivar la socialización de los niños e involucrar a los adultos. Esto incluyó caminatas, campamentos, cenas de camaradería. Tales actividades propiciaron la integración de vecinos de diferentes barrios y hasta de distintas instituciones. De hecho, la mayoría de esas instancias tenía lugar en la sede de otras entidades barriales con las cuales los clubes entablaban relaciones. Los encuentros de este tipo se extendían más allá del plano asociativo y reforzaban los vínculos vecinales. De esta manera, los clubes se constituyeron como espacios participativos atravesados por afinidades y redes de sociabilidad, con relaciones relativamente igualitarias, que conllevaban una serie de responsabilidades asumidas por sus miembros (Reyna, 2011). Allí se difundieron valores democráticos y los sectores populares pudieron interactuar y visibilizar su presencia en el espacio público.

Gracias a los lazos de solidaridad generados a partir de la organización comunitaria, la AFIB hizo frente a los efectos más graves de la crisis hiperinflacionaria que azotó a la Argentina a finales de los años ochenta. En este contexto, la Asociación organizó campeonatos de carácter regional, nacional e internacional que se disputaron en la ciudad. Los documentos de que disponemos dan cuenta de la organización del Primero y el Segundo Torneo Internacional de Fútbol Infantil, realizados en 1989 y 1990 (AFIB, 1989-1990), a los que la AFIB invitó a participar a equipos provenientes de otros puntos de la Argentina y de toda Sudamérica («Excelente inicio fútbol, 1989»; «Fútbol infantil», 1989). Los visitantes eran hospedados en casas de vecinos o en hoteles a través de las 
gestiones que realizaba la Asociación de Fútbol Infantil. La organización de estos certámenes era llevada adelante con voluntarismo y muchas veces con el único apoyo económico de pequeños comercios.

A la par de la celebración de estas competiciones en Bariloche, los equipos de la ciudad realizaron numerosos viajes para disputar torneos en otros lugares del país, lo cual era posible a través de las redes asociativas que la AFIB tejía con instituciones similares en el resto de la Argentina, así como también en otros países de la región. De esta manera, conjuntos de Bariloche viajaron a las provincias de Santa Fe, Córdoba, Chubut, Neuquén, Buenos Aires, La Pampa y localidades del sur de Chile, entre otros sitios. La propuesta de la Asociación era llevar equipos foráneos a Bariloche y, a la vez, fomentar que los niños de los barrios pudieran conocer otros puntos del país. La realización de estos viajes estaba ligada a los objetivos de formación y contención social planteados por la Asociación.

En el mismo sentido, contamos con registros de un proyecto de «integración provincial» establecido en 1992 entre la AFIB y la Dirección General de Deportes de Río Negro, orientado a generar intercambios deportivos entre las distintas regiones de la provincia («Salidas preparativos, 1993»). Con el mismo propósito de generar integración se dio la conformación de selecciones infantiles de Bariloche que disputaban campeonatos de alcance regional y nacional. ${ }^{12}$ Sin embargo, estos proyectos generalmente tuvieron poca continuidad, ya que su conformación se vio limitada por la precariedad material de la AFIB.

\section{La intervención estatal y los vínculos interinstitucionales}

Los apoyos del gobierno municipal a la actividad del fútbol infantil estuvieron presentes a lo largo del período seleccionado. Sin embargo, en general se redujeron a aportes aislados, poco sistemáticos o promesas de colaboración. De acuerdo con los testimonios, se trató concretamente de algunos subsidios eventuales destinados a transporte y alojamiento para los viajes de los equipos. En los documentos de la AFIB se encuentran referencias a otro tipo de intervención estatal con las características mencionadas, como, por ejemplo, la promesa de comprar elementos deportivos, realizar cursos de capacitación para los técnicos y entregar subsidios para el desarrollo de algún torneo específico. Una medida significativa en términos económicos fue la eximición del pago de cargas impositivas municipales para los — pocos— clubes que contaban con un predio 
y una sede. ${ }^{13}$ Sin embargo, ello da cuenta de una pauta: los apoyos de los gobiernos provinciales y municipales solían limitarse a las instituciones que poseían un terreno. Asimismo, la ayuda gubernamental se mostró inconclusa en relación con la gestión de espacios propios para el fútbol infantil (AFIB, 1987k, 1987l) y se redujo a la cesión de horas de uso del estadio y los gimnasios municipales.

Sin aportes del Estado y sin suficientes fuentes de ingresos, la actividad se sostenía con voluntarismo a través de las tareas desarrolladas por los vecinos y los dirigentes. Asimismo, los clubes buscaron entablar vínculos con otras instituciones barriales. Algunos se constituyeron como subcomisiones de deportes de las juntas vecinales, lo que les permitió obtener personería jurídica para poder integrar la AFIB. Ejemplo de ello fueron algunos clubes pertenecientes a los barrios populares de la ciudad $-\mathrm{y}$ bautizados de igual modo que estos-, tales como el Club Barrio Lera, el Club 10 de Diciembre, el Club Barrio Arrayanes y el Club San Francisco II y III. La mayoría de estas instituciones surgieron en la segunda mitad de la década de 1980, de forma casi simultánea a la creación de las juntas vecinales. Los clubes con estas características tenían un anclaje identitario barrial y recibían a niños provenientes de su entorno espacial más cercano, pero también de otros sectores de la ciudad e incluso de las localidades próximas. De esta manera, el fútbol reforzó las interacciones intra e interurbanas y permitió a diversos individuos participar en esferas que antes eran inaccesibles para ellos.

La relación entre las entidades barriales se concretaba en un conjunto de actividades y eventos destinados a obtener fondos. Además, el nexo podía resultar beneficioso para realizar gestiones ante la Municipalidad. Sin embargo, los vínculos institucionales no carecieron de conflictos y diferencias de intereses, lo que en ocasiones resultó una limitación para el crecimiento de las entidades deportivas.

\section{Neoliberallismo y el finall de la AFIB}

Durante los años noventa, las políticas económicas neoliberales implantadas en Argentina trajeron consigo la reducción del gasto público y el desmantelamiento de las funciones protectoras del Estado. Una de las consecuencias fue el aumento de la desocupación y los niveles de pobreza de la población. Estos efectos, junto con la valorización del paradigma del individualismo, generaron procesos de desciudadanización y la desarticulación de lazos sociales. El impacto de las políticas neoliberales más profundas se vio amortiguado en Río Negro durante la primera mitad de la década, debido al mantenimiento de un Estado providencialista sostenido por los sucesivos gobiernos radicales 
desde el retorno de la democracia, aunque, aun así, el período estuvo marcado por las crisis fiscales en la provincia (Camino Vela, 2015). En este marco, la problemática de las organizaciones populares se vio agravada en Bariloche por los procesos de segregación socioterritorial en los barrios, producto de la falta de planificación urbana que marginó a los sectores de bajos recursos y así dio lugar a grandes contrastes sociales en la ciudad. La fragmentación contribuyó al debilitamiento de las acciones colectivas y originó múltiples situaciones de marginalidad y vulnerabilidad —entendida como manifestación de la falta de integración social y la fragilidad de las redes de solidaridad- (Núñez y Fuentes, 2008).

Las políticas neoliberales repercutieron negativamente en los clubes barriales. Por eso, la década de 1990 es señalada como un momento de decadencia de estas instituciones (Zambaglione et al., 2013). Como resultado de la reducción del gasto público, el deporte social quedó relegado frente al deporte de alto rendimiento y la realización de eventos de envergadura nacional e internacional. Durante estos años se desarticularon muchos vínculos sociales que habían hecho posible la organización comunitaria e institucional después del retorno de la democracia, lo que también se manifestó en el ámbito del fútbol infantil barilochense. De acuerdo con los testimonios de algunos exdirigentes, uno de los principales problemas de la época fue el alejamiento de los socios y el escaso involucramiento de los vecinos a la hora de colaborar con las actividades de los clubes. En efecto, los dirigentes debían costear con recursos propios el desarrollo deportivo. Esto se comprende de manera situada, ya que para muchas personas el compromiso con las entidades quedaba en un segundo plano, dadas las agobiantes necesidades económicas.

Como consecuencia se produjo la desaparición de algunos de los clubes que habían integrado originalmente a la AFIB, así como la emergencia de otros que experimentaron constantes vaivenes en su estabilidad institucional. Entrados los años noventa, la cantidad de clubes miembros de la Asociación fue aumentando y diversificándose - a la par de dicha fragmentación urbana-, lo que amplió la participación de equipos de toda la ciudad. Varios de ellos eran representativos de los barrios populares, que para poder participar recurrieron a alianzas con las juntas vecinales. Por otra parte, fue cada vez más recurrente la presencia de combinados provenientes de localidades cercanas. Sin embargo, muchos de los nuevos clubes contaban con pocas categorías y su participación en los campeonatos era intermitente. En general, eran «organizaciones laxas» con poca o nula infraestructura (Reyna, 2011).

La falta de formación en términos dirigenciales y la ausencia de proyectos deportivos de largo plazo fueron factores que limitaron el crecimiento y el desarrollo de las instituciones. En este sentido corrieron la centralización de los procesos de toma de decisión en unas pocas personas y la falta de renovación de las comisiones de los clubes, por ausencia de asambleas y omisión de los procesos electorales establecidos en los reglamentos, lo que muchas veces generó conflictos internos. 
El 10 de octubre de 1994 la Asociación de Fútbol Infantil Bariloche se fusionó con la Liga de Fútbol Bariloche. La fusión apuntó al fortalecimiento institucional y a favorecer el deporte para niños mediante una mayor articulación con las categorías juveniles («Fusión liga», 1994). La situación previa a esta decisión era difícil para la AFIB en términos económicos y organizativos. ${ }^{14}$ Las complicaciones eran causadas principalmente por las deudas en las cuotas mensuales que debían aportar los clubes, muchos de los cuales se encontraban en condiciones igualmente críticas. Ante esto, la fusión con LIFUBA resultaba prometedora en lo que respecta a las finanzas de la AFIB. Sin embargo, en los años posteriores observamos continuidades en cuanto a las dificultades materiales de los clubes y la falta de apoyos estatales.

Por otra parte, a lo largo de la década desaparecieron numerosos espacios públicos empleados como campos de juego en toda la ciudad, en los cuales se construyeron instituciones, planes de vivienda o se dieron tomas ilegales de tierras. Esto fue resultado del auge de los intereses inmobiliarios — con aval del Estado - por sobre las demandas de tierra por los sectores populares, lo que profundizaba una tendencia que recorrió el último cuarto del siglo XX.

\section{Reflexiones finales}

En este artículo reconstruimos la institucionalización del fútbol infantil en Bariloche y analizamos las sociabilidades desarrolladas en torno a la práctica deportiva. Abordamos las particularidades de la AFIB con el objetivo de aportar una reflexión respecto de la conformación y el funcionamiento de las instituciones populares en la localidad y el estudio de las interacciones barriales en el tiempo libre. Ello desde una perspectiva que vincula las sociabilidades deportivas con las dinámicas sociales y vecinales entre los años posteriores al retorno de la democracia en Argentina, en 1983, y la implantación de políticas neoliberales, en los años noventa. De esta manera, intentamos profundizar, desde el estudio del fútbol y sus instituciones, en el proceso de crecimiento urbano en Bariloche y el impacto de las crisis económicas y sociales sobre los sectores populares. De modo más amplio, esperamos que este avance de investigación aporte a los análisis acerca de los clubes de fútbol en la historia reciente argentina en clave local y barrial, complementando y complejizando las miradas enfocadas en las instituciones más grandes del país dedicadas al deporte profesional. 
Durante el período abordado se dio la emergencia de numerosas experiencias asociativas vecinales. La AFIB y la mayoría de los clubes que formaron parte de ella surgieron en el marco de un proceso de organización comunitaria a partir de la segunda mitad de los años ochenta, favorecido por la restauración de la institucionalidad democrática y motivado por las demandas de la población de las barriadas en crecimiento por contar con espacios de recreación y socialización. Estas entidades propiciaron la interacción vecinal y la participación ciudadana, así como también la difusión de valores democráticos en un marco de relaciones relativamente igualitarias. La integración barrial resulta significativa teniendo en cuenta que en el último cuarto del siglo XX se profundizaron los procesos de segregación socioespacial, lo que dio lugar a grandes contrastes dentro de la ciudad. Específicamente, la Asociación de Fútbol Infantil mantuvo un discurso y acciones que apuntaron a generar contención social frente a las problemáticas que afectaban a las familias de los barrios populares de Bariloche.

Sin embargo, el período en cuestión también nos permite ver las limitaciones que debieron afrontar dichas instituciones, las cuales jugaron en contra de la consolidación de las experiencias asociativas. Esto se observa en las problemáticas económicas y organizativas que atravesaron la vida institucional de la AFIB, así como la precariedad material de los clubes. A finales de los años ochenta, estas entidades sufrieron el impacto de la crisis hiperinflacionaria que azotó a la Argentina. Posteriormente, desde principios de la década de 1990, el paradigma del neoliberalismo propició la desarticulación de lazos sociales, junto con los efectos de las políticas económicas. A estas características del contexto a escala macro sumamos, de modo transversal, el voluntarismo con que eran sostenidas estas instituciones — de forma casi exclusiva-, en parte debido a la escasez de políticas públicas en beneficio de las organizaciones deportivas barriales en la ciudad. En general, los apoyos gubernamentales en favor del fútbol infantil se caracterizaron por ser discretos, aislados y poco sistemáticos.

Frente a este panorama, los dirigentes de la AFIB y los clubes impulsaron distintas actividades destinadas a desarrollar la estructura institucional del deporte. Para ello buscaron entablar vínculos con otras entidades de su entorno barrial, como las juntas vecinales. Muchas de las relaciones construidas trascendían el plano institucional y reforzaban los vínculos vecinales en encuentros más o menos espontáneos en ámbitos públicos y privados. De este modo, los clubes se constituyeron como espacios de sociabilidad deportiva y popular, generadores de sentimientos de pertenencia, que favorecieron la participación y la interacción vecinal de los sectores populares en el espacio público. Asimismo, en la vida cotidiana, motivaron la construcción de diversas relaciones institucionales con otras organizaciones similares o diferentes, en el medio local o fuera de la ciudad. En este punto, el fútbol fue el elemento articulador a través del cual se construyeron redes de encuentro en distintos lugares de la ciudad que funcionaron como ámbitos unificadores que congregaban a vecinos de sectores alejados entre sí. De 
esta manera, el deporte favoreció la apropiación y la producción del espacio urbano en reuniones que fomentaban la integración vecinal.

Si bien el fútbol no perdió ese lugar, durante los años noventa la profundización de los procesos de fragmentación y segregación socioespacial contribuyó a debilitar las acciones colectivas y dio lugar a múltiples situaciones de marginalidad y vulnerabilidad. La desarticulación de los vínculos sociales que habían hecho posible la organización comunitaria después del retorno de la democracia repercutió negativamente en los clubes. Esto se observa en la fragilidad de las estructuras institucionales y en el hecho de que la actividad era sostenida muchas veces con recursos propios de los dirigentes.

Por otra parte, la experiencia de la AFIB nos lleva a pensar en las particularidades locales de la sociabilidad deportiva y el desarrollo de las instituciones frente a los procesos de globalización y mercantilización del deporte en el mundo y en las grandes ciudades de la Argentina. Bariloche es una ciudad que cuenta con reconocimiento internacional, recibe turistas de todo el país y de distintas nacionalidades. Sin embargo, esta inserción en la economía mundial no permeó el devenir del fútbol local, ya que las sociabilidades y el devenir institucional tuvieron que ver más con el amateurismo y el voluntarismo que con la comercialización del deporte-espectáculo y la popularización masiva de la actividad. Incluso la organización de torneos nacionales e internacionales se dio a pulmón, priorizando la formación y la contención social a través del deporte en su fase recreativa. La institucionalización del fútbol infantil barilochense fue un proceso tardío que se concretó en el contexto finisecular, marcado por la precariedad material de los clubes y las iniciativas de las comunidades barriales. Esta realidad puede asociarse a la dinámica del crecimiento urbano, la heterogeneidad social y las dificultades del Estado municipal para instalar su presencia en las barriadas periféricas o populares.

\section{Fuentes}

Asociación del Fútbol Infantil Bariloche. (1987a). Acta de reunión de dirigentes y delegados de los clubes de la AFIB. [Documento]. Archivo privado.

Asociación del Fútbol Infantil Bariloche. (1987b). Nota informativa №1 enviada a los clubes locales. [Documento]. Archivo privado.

Asociación del Fútbol Infantil Bariloche. (1987c). Reglamentación estatutaria de la AFIB. [Documento]. Archivo privado.

Asociación del Fútbol Infantil Bariloche. (1987d, 22 de abril). Acta de reunión de dirigentes y delegados de los clubes de la AFIB. [Documento]. Archivo privado.

Asociación del Fútbol Infantil Bariloche. (1987e, 9 de junio). Acta de reunión de dirigentes y delegados de los clubes de la AFIB. [Documento]. Archivo privado. 
Asociación del Fútbol Infantil Bariloche.(1987f, 16 de junio). Acta de reunión de dirigentes y delegados de los clubes de la AFIB. [Documento]. Archivo privado.

Asociación del Fútbol Infantil Bariloche. (1987g, 7 de abril). [Nota del club Independiente dirigida a la $A F I B]$. [Documento]. Archivo privado.

Asociación del Fútbol Infantil Bariloche. (1987h, 9 de mayo). Acta de reunión de dirigentes y delegados de los clubes de la AFIB. [Documento]. Archivo privado.

Asociación del Fútbol Infantil Bariloche. (1987i, 28 de abril). Acta de reunión de dirigentes y delegados de los clubes de la AFIB. [Documento]. Archivo privado.

Asociación del Fútbol Infantil Bariloche. (1987j, 6 de octubre). Acta de reunión de dirigentes y delegados de los clubes de la AFIB. [Documento]. Archivo privado.

Asociación del Fútbol Infantil Bariloche. (1987k, 12 de mayo). Acta de reunión de dirigentes y delegados de los clubes de la AFIB. [Documento]. Archivo privado.

Asociación del Fútbol Infantil Bariloche. (1987l,19 de mayo). Acta de reunión de dirigentes y delegados de los clubes de la AFIB. [Documento]. Archivo privado.

Asociación del Fútbol Infantil Bariloche. (1989, 7 de febrero). [Nota de la AFIB dirigida a la Asociación Barilochense de Árbitros (ABA)]. [Documento]. Archivo privado.

Asociación del Fútbol Infantil Bariloche. (1989-1990). [Actas de reuniones de dirigentes y delegados de los clubes de la AFIB]. [Documento]. Archivo privado.

Asociación del Fútbol Infantil Bariloche. (1990, 20 de marzo). [Nota de la AFIB dirigida al Colegio de Árbitros del Fútbol Libre]. [Documento]. Archivo privado.

Club 9 de Julio. (1990, 21 de junio). [Nota enviada a la Radio del Pueblo]. [Documento]. Archivo privado.

Club Alas Argentinas. (1990, 13 de agosto). [Nota dirigida a la AFIB]. [Documento]. Archivo privado.

Club Don Bosco. (1989, 17 de enero). [Nota al programa de radio Tribuna deportiva]. [Documento]. Archivo privado.

Club Estudiantes Unidos. (1990, 19 de junio). [Nota dirigida a la AFIB]. [Documento]. Archivo privado.

Club Virgen del Carmen. (1987, 7 de noviembre). [Nota dirigida a la AFIB]. [Documento]. Archivo privado.

Escuela de Fútbol de Martín Güemes. (1990, 20 de noviembre). [Nota dirigida a la AFIB]. [Documento]. Archivo privado.

Escuela de Fútbol Infantil de Tiro Federal. (1990, 13 de agosto). [Nota dirigida a la AFIB]. [Documento]. Archivo privado.

Excelente inicio del fútbol infantil. (1989, 14 de febrero). Diario Río Negro. Fútbol infantil: Torneo internacional. (1989, febrero). Visión Deportes (Bariloche).

La fusión de la liga con la AFIB. (1994, octubre). Tribuna Deportiva.

Liga de Fútbol de Bariloche. (1979). Estatuto. [Documento]. Archivo privado. Salidas y preparativos del club Alas Argentinas. (ca 1993). Diario Bariloche. 


\section{Referencias bibliográficas}

Camino Vela, F. (2015). La provincia de Río Negro entre 1983 y 2003: Predominio radical bajo diferentes modelos. Revista POSTData, 19, 2.

Chiappe, M. C. (2019). Lo que se aprende con el cuerpo no se olvida más: El deporte como vehículo de reproducción ideológica en la región del Nahuel Huapi, 1930 1955 (Tesis de maestría). Universidad Nacional de Quilmes, Buenos Aires.

Cucó i Giner, J. (2008). Sociabilidades urbanas. Ankulegi, (12), 65-82.

Escalera, J. (2000). Sociabilidad y relaciones de poder. Kairos: Revista de Temas Sociales, (6).

Groisman, F. (2010). La persistencia de la segregación residencial socioeconómica en Argentina. Estudios Demográficos y Urbanos, 25(2), 429-460.

Hang, J. (2018). Política y sociabilidad en un club social y deportivo de la ciudad de La Plata (Tesis de doctorado). Universidad Nacional de La Plata, La Plata.

Ignacio, B. (2013). La dinámica Barrio-Club y su articulación con las políticas estatales. Ponencia presentada en XIV Jornadas Interescuelas/Departamentos de Historia, Mendoza. Recuperado de https://cdsa.aacademica.org/000-010/979.pdf.

Lvovich, D., y Rodríguez, L. G. (2011). La gendarmería infantil durante la última dictadura. Quinto Sol, 15(1).

Matossian, B. (2014). Proceso de expansión urbana, actores y desigualdades. Revista Estudios Sociales Contemporáneos, 10, 60-68.

Matossian, B. (2016). Fragmentación urbana y asociaciones vecinales en San Carlos de Bariloche, Patagonia-Argentina (1983-2015). Papeles de Geografía, (62), 64-76.

Navarro, J. (2006). Sociabilidad e historiografía: Trayectorias, perspectivas y retos. Saitabi, Revista de la Facultat de Geografia i História, (56), 99-119.

Neiman, G., y Quaranta, G. (2006). Los estudios de caso en la investigación sociológica. En I. Vasilachis de Gialdino (comp.), Estrategias de investigación cualitativa. Buenos Aires: Gedisa.

Nestares, J. (2017). Fútbol y sectores populares: La historia de ADEFUL. Neuquén: Educo.

Núñez, P., y Fuentes, R. (2008). Formas de participación en procesos democráticos recientes: Sociedad civil en San Carlos de Bariloche. Recerca: Revista de Pensamient i Anàlisi, (8), 193-213.

Rein, R. (2015). La cancha peronista: Fútbol y política (1946-1955). San Martín: UNSAM Edita.

Reyna, F. (2011). La formación y difusión de los clubes de fútbol en Córdoba (19001920): Espacios de sociabilidad y prácticas asociativas. Ponencia presentada en 3ras. Jornadas Nacionales de Historia Social, La Falda, Córdoba.

Zambaglione, D., Fitipaldi, G., Levoratti, A., Maiori, M., y Cañueto, M. (2013). Los clubes de barrio: Deporte social y recreación en un espacio de inclusión social. Revista Impetu, 7(2), 35-41. 